\title{
Photoluminescent Colloidal Nanohelices Self-Assembled from CdSe Magic-Size Clusters via Nanoplatelets
}

\author{
Yuanyuan Liu, ${ }^{\dagger}$ Nelson Rowell, ${ }^{\ddagger}$ Maureen Willis, ${ }^{\S}$ Meng Zhang, ${ }^{\dagger}$ \\ Shanling Wang," Hongsong Fan, $\perp$ Wen Huang," Xiaoqin Chen*,§, Kui Yu*, ${ }^{*}, \perp$,
}

'Institute of Atomic and Molecular Physics, Sichuan University, 610065 Sichuan, P. R. China

${ }^{\ddagger}$ Metrology Research Centre, National Research Council Canada, Ottawa, Ontario K1A 0R6, Canada

§School of Physical Science and Technology, Sichuan University, 610065, P. R. China

"Analytical \& Testing Center, Sichuan University, 610065 Sichuan, P. R. China

${ }^{\perp}$ Engineering Research Center in Biomaterials, Sichuan University, 610065 Sichuan, P. R. China

\#Laboratory of Ethnopharmacology, West China School of Medicine, Sichuan University, 610065 Sichuan, People's Republic of China

$\varrho^{@}$ State Key Laboratory of Polymer Materials Engineering, Sichuan University, 610065 Sichuan, P. R. China

*X.C. E-mail: $\underline{x q c h e n @ s c u . e d u . c n} \quad *$ K. Y. E-mail: kuiyu@scu.edu.cn. 
Table of Contents

Experimental Methods

Table S1 Summary of the preparation of CdSe nanostructures addressed.

Figure S1-1 Three additional TEMimages for Dispersion b of Figure 1. 5

Figure S1-2 Optical and TEM study of dispersions with different sonication periods. 6

Figure S1-3 Optical and TEM study of dispersions containing different amounts of HDA. 8

Figure S2-1 Repeated Figure 2study. 9

Figure S2-2 Optical and TEM study of one purified sample in $\mathrm{CHCl}_{3}$ containing OLA or OLA. 10

Figure S2-3 Optical spectra and TEM images for one Tol-EtOH purified sample in Tol. 11

Figure S2-4 Optical properties of one purified sample in Tol-HDA and $\mathrm{CHCl}_{3}-\mathrm{HDA}$ dispersions 12

Figure S3 Optical and TEM study of one Tol- $\mathrm{CH}_{3} \mathrm{CN}$ purified sample in Tol. 13

Figure S4-1 TEM images of two samples incubated 7 and 38 days. 14

Figure S4-2 Optical spectra collected for Dispersions a and b of Figure 4. 15

Figure S5 Polarized emission of CdSe ODMSCs, 2D NPLs and nanohelices. 16 


\section{Experimental Methods}

\section{Chemicals}

All chemicals are commercially available and were used as received, including cadmium oxide (CdO, Aldrich), selenium powder (Se, 99.99\%, Alfa Aesar), 1-octadecene (ODE, 90.0\%, Aldrich), myristic acid (MA, 99.0\%, Aldrich), acetic acid (HOAc, 99.5\%, Chengdu Kelong), octylamine (OTA, 99.0\%, Aldrich), oleylamine (OLA, 70.0\%, Aldrich), hexadecylamine (HDA, 90.0\%, Aldrich), ethanol (EtOH, 95\%, Chendu Kelong), acetonitrile $\left(\mathrm{CH}_{3} \mathrm{CN}, 99.8 \%\right.$, Aldrich), chloroform $\left(\mathrm{CHCl}_{3}\right.$, 99.0\%, Chengdu Kelong), and toluene (Tol, 99.5\%, Chengdu Kelong),

\section{Stock solution preparation and the synthesis of CdSe OD MSCs}

For our $\mathrm{Cd}(\mathrm{MA})_{2}$ stock solution, cadmium oxide ( $\left.\mathrm{CdO}, 0.5136 \mathrm{~g}, 4.00 \mathrm{mmol}\right)$, myristic acid (MA, $\mathrm{C}_{13} \mathrm{H}_{27} \mathrm{COOH}, 2.0097 \mathrm{~g}, 8.80 \mathrm{mmol}$ ), and 1-octadecene (ODE, $17.4767 \mathrm{~g}$ ) were added to a $50 \mathrm{~mL}$ three-necked round-bottom flask, which was equipped with a condenser. The mixture was degassed three times and placed under vacuum for 30 min until no bubbles were observed while stirring at room temperature. Then, the reaction temperature was increased to $240{ }^{\circ} \mathrm{C}$ under $\mathrm{N}_{2}$. CdO dissolved completely and a clear solution was obtained eventually after $60 \mathrm{~min}$. The mixture was then cooled down to $110{ }^{\circ} \mathrm{C}$ and placed under vacuum for two hours to remove the $\mathrm{H}_{2} \mathrm{O}$ produced during reaction. The mixture was then cooled to room temperature for storage.

To synthesize CdSe OD MSCs that exhibit an absorption doublet at 433/461 nm, the Cd(MA)2 stock solution $(3.0000 \mathrm{~g}, \mathrm{Cd}=0.60 \mathrm{mmol})$, Se powder $(0.0118 \mathrm{~g}, 0.15 \mathrm{mmol})$ and ODE $(0.9882 \mathrm{~g})$ with a total weight of $4.0000 \mathrm{~g}$ were added to a $50 \mathrm{~mL}$ three-necked round-bottom flask. The mixture was degassed for three times and placed under vacuum while stirring at room temperature (RT) for 30 min till no bubbles were observed. The reaction mixture was then heated to $120^{\circ} \mathrm{C}$ and kept at this temperature for 2 hours. The reaction batch was connected to $\mathrm{N}_{2}$ afterwards, to which a mixture of $0.3000 \mathrm{~g}$ acetic acid $(5.00 \mathrm{mmol})$ and $0.7000 \mathrm{~g}$ ODE was added dropwise. The reaction mixture was then heated to $220{ }^{\circ} \mathrm{C}$ and reacted at this temperature for $15 \mathrm{~min}$ to obtain the CdSe sample.

For sample purification, $15 \mu \mathrm{L}$ of our as-synthesized sample was dispersed in Tol (2.0 mL), with 
the dropwise addition of $1.0 \mathrm{~mL}$ of $\mathrm{EtOH}$ or $\mathrm{CH}_{3} \mathrm{CN}$. The resulting dispersion was then centrifuged on a Shuke Centrifuge TG-16S at a speed of $9000 \mathrm{rpm}$ for $2 \mathrm{~min}$. After centrifugation, the supernatant was removed, and the resulting precipitate was subjected to the same process for another two times.

\section{Characterization}

One as-synthesized or purified sample $(15 \mu \mathrm{L})$ was dispersed in Tol $(3.0 \mathrm{~mL})$ or $\mathrm{CHCl}_{3}(3.0 \mathrm{~mL})$ containing HDA (such as $0.010 \mathrm{~g}$ ). Sonication was carried out on a KH5200E sonicator at room temperature, with frequency and power of $40 \mathrm{~Hz}$ and $200 \mathrm{~W}$.

For the optical property characterizations, UV-vis absorption spectra were collected on a Cary 5000 spectrometer from 300 to $600 \mathrm{~nm}$ with an interval of $1 \mathrm{~nm}$. Photoluminescence spectra were collected on a Horiba Fluoromax-4 spectrometer with an excitation wavelength of either 350 or $410 \mathrm{~nm}$, as illustrated in corresponding figures. The emission spectra were collected from 360 to $600 \mathrm{~nm}$ (when excited at $350 \mathrm{~nm}$ ) or 420 to $600 \mathrm{~nm}$ (when excited at $410 \mathrm{~nm}$ ) with an interval of $1 \mathrm{~nm}$.

For the transmission electron microscopy (TEM) measurements, the dispersion sample was drop casted on a cooper grid, which placed in a fume-hood for the evaporation of solvent. The images were taken on FEI Tecnai G2 F20 S-TWIN and JEM-2100 Plus (Sichuan University). 
Table S1. Summary of the preparation of CdSe OD MSCs, 2D NPLs and nano-helices addressed.

\begin{tabular}{|c|c|c|c|c|}
\hline Figure & Purification & Dispersion & Sonication & \\
\hline Fig 1a & No & 3.0Tol & No & MSCs \\
\hline Fig $1 b$ & No & 3.0Tol - 0.010g HDA & $5 \mathrm{~min}$ & Nano-helices \\
\hline Fig S1-2A a & No & 3.0Tol - 0.010g HDA & No & NPLS \\
\hline $\begin{array}{l}\text { Figs S1-2A } \\
b / c\end{array}$ & No & 3.0Tol - 0.010g HDA & $\begin{array}{l}5 / 120 \\
\min \end{array}$ & Nano-helices \\
\hline Fig S1-2B & No & 3.0Tol - 0.010g HDA & $30 \mathrm{~min}$ & Nano-helices \\
\hline Fig S1-3 & No & $\begin{array}{l}\text { 3.0Tol - } \\
\text { 0.005/0.020/0.040g HDA }\end{array}$ & $30 \mathrm{~min}$ & Nano-helices \\
\hline $\begin{array}{l}\text { Figs 2a, S2- } \\
1 \mathrm{a}\end{array}$ & $1 \mathrm{EtOH}-2 \mathrm{Tol}$ & 3.0Tol & No & NPLS \\
\hline $\begin{array}{l}\text { Figs } 2 b, 52- \\
1 b\end{array}$ & 1EtOH-2Tol & $3.0 \mathrm{CHCl}_{3}-0.010 \mathrm{~g} \mathrm{HDA}$ & $30 \mathrm{~min}$ & Nano-helices \\
\hline Fig S2-2 & $1 \mathrm{EtOH}-2 \mathrm{Tol}$ & $\begin{array}{l}3.0 \mathrm{CHCl}_{3}-0.010 \mathrm{~g} \\
\text { OTA/OLA }\end{array}$ & $30 \mathrm{~min}$ & Nano-helices \\
\hline Fig S2-3 & $\begin{array}{l}\text { 1EtOH-2Tol } \\
\text { (38-day incubated) }\end{array}$ & 3.0Tol & $30 \mathrm{~min}$ & Nano-helices \\
\hline Fig 3a, S3 & $1 \mathrm{CH}_{3} \mathrm{CN}-2 \mathrm{Tol}$ & 3.0Tol & No & MSCs \\
\hline \multicolumn{5}{|l|}{ Fig $3 b$} \\
\hline & $1 \mathrm{CH}_{3} \mathrm{CN}-2 \mathrm{Tol}$ & $3.0 \mathrm{CHCl}_{3}-0.010 \mathrm{~g} \mathrm{HDA}$ & $30 \mathrm{~min}$ & Nano-helices \\
\hline Fig 4a & $\begin{array}{l}\text { No } \\
\text { (38-day incubated) }\end{array}$ & 3.0Tol & No & MSCs, NPLs \\
\hline Fig 4b & $\begin{array}{l}\text { No } \\
\text { (38-day incubated) }\end{array}$ & 3.0Tol & $30 \mathrm{~min}$ & Nano-helices \\
\hline Fig S4-1 & $\begin{array}{l}\text { No (7-day or } \\
\text { 38-day incubated) }\end{array}$ & 3.0Tol - 0.010g HDA & None & $\begin{array}{l}\text { NPLs, Nano- } \\
\text { helices } \\
\text { Nano-helices }\end{array}$ \\
\hline
\end{tabular}




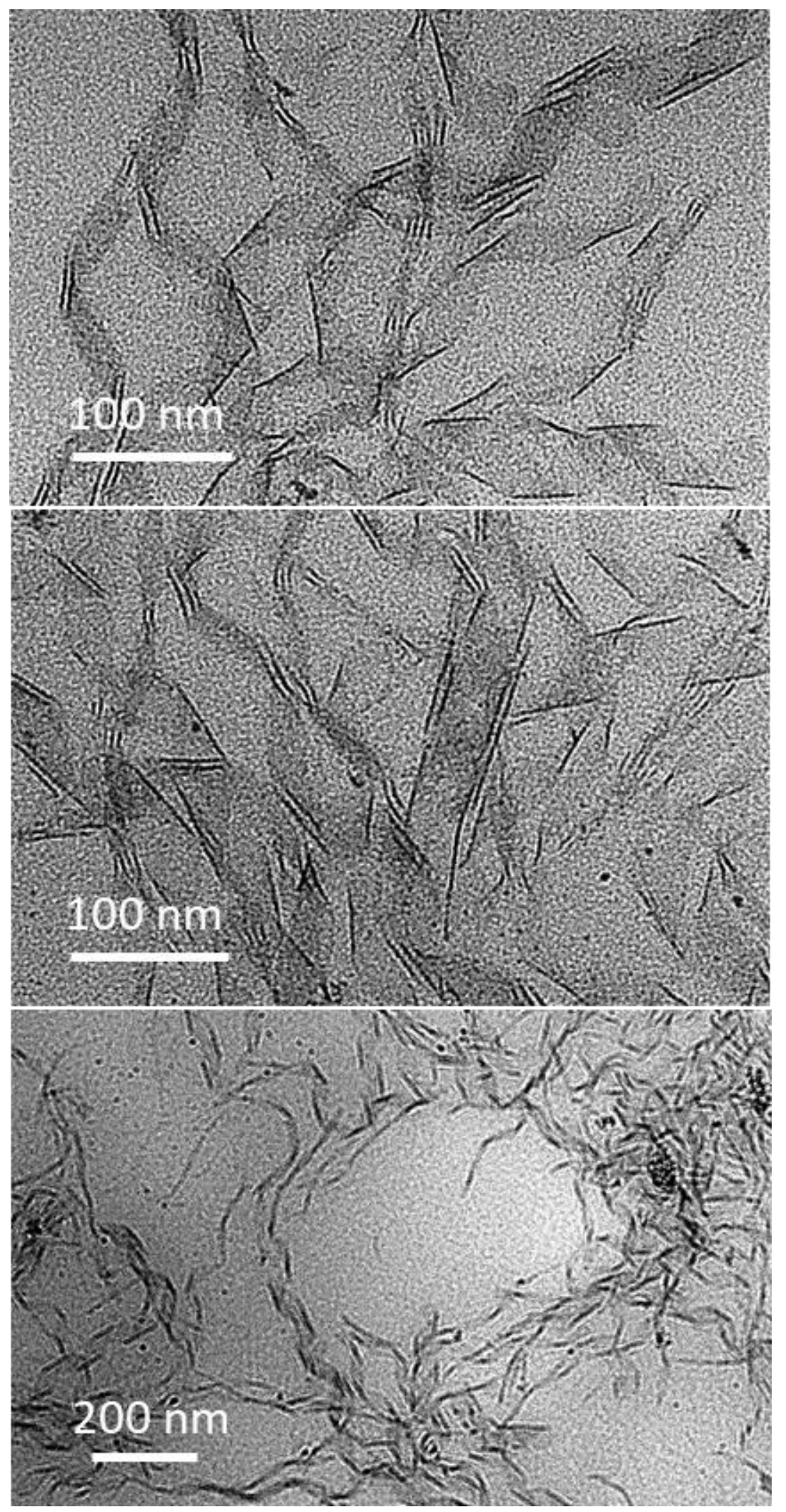

Figure S1-1. Additional TEM images for Dispersion b of Figure 1. 

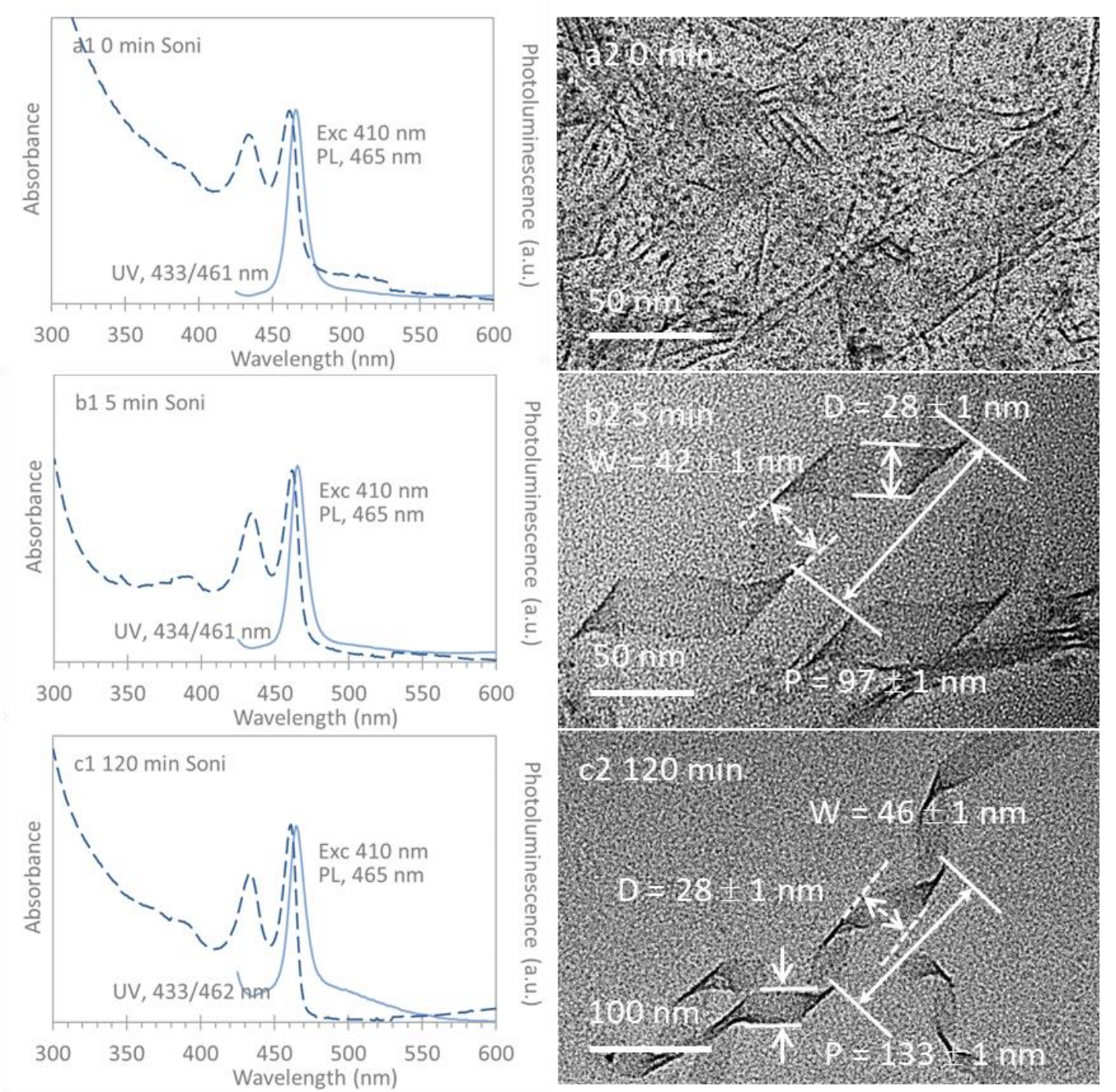

Figure S1-2A. Normalized absorption (dashed traces, left $y$ axis) and emission (solid traces, right $y$ axis) spectra and TEM images of one as-synthesized sample $(15 \mu \mathrm{L})$ in $3.0 \mathrm{~mL}$ Tol 0.010 g HDA, with 0 (a), 5 (b) and 120 (c) min sonication. Without sonication, 2D NPLs together with OD MSCs are observed (a2); with sonication, 2D helical nanostructures are formed (b2 and c2). There is large experimental window for the sonication process to facilitate the formation of the helical nanostructure, under our experimental conditions. 


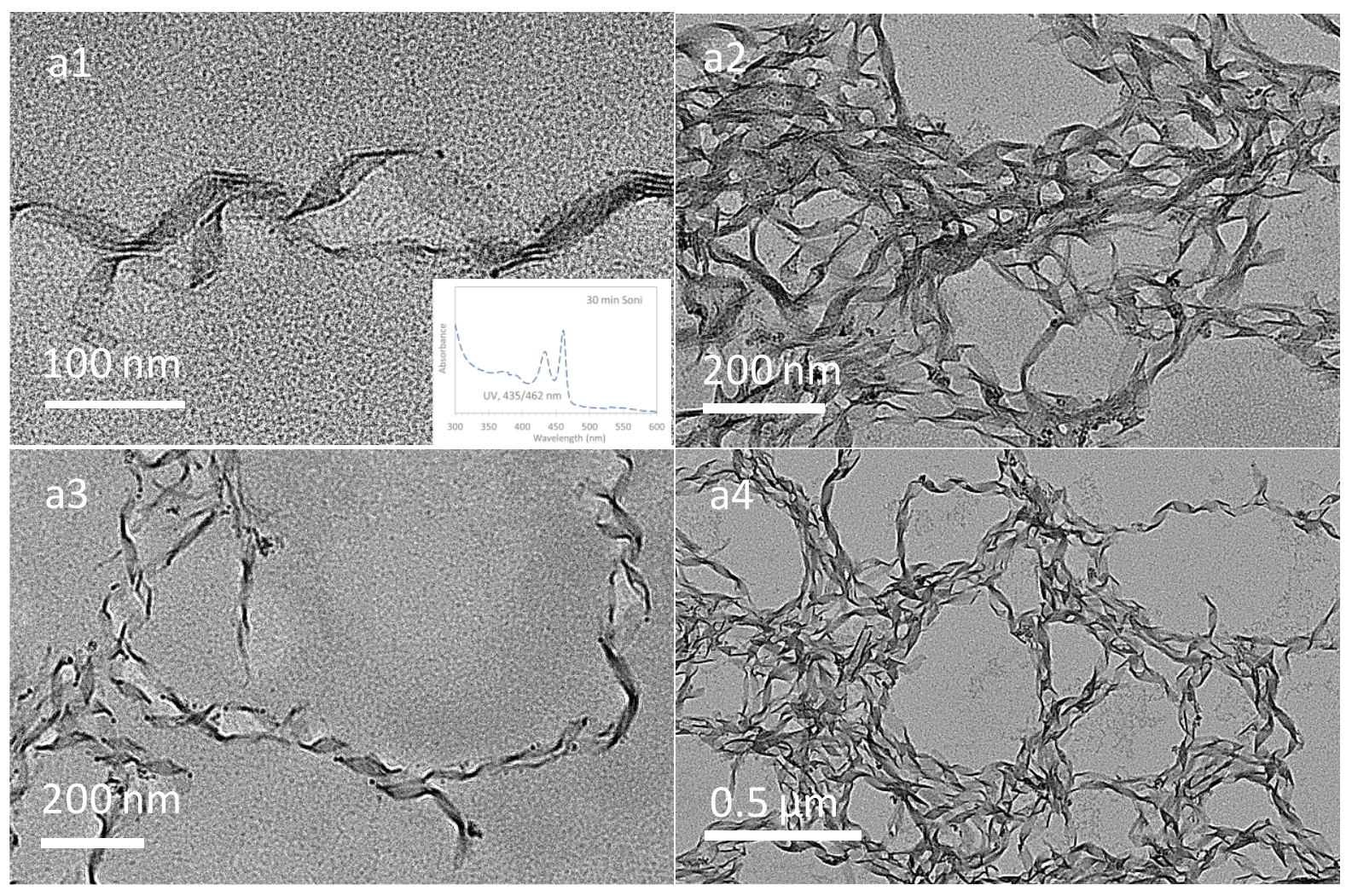

Figure S1-2B. TEM images of one as-synthesized sample $(15 \mu \mathrm{L})$ in $3.0 \mathrm{~mL}$ Tol $-0.010 \mathrm{~g}$ HDA, with 30 min sonication. Clearly, 2D helical nanostructures are formed, and the inset in a1 contains their absorption spectrum. 

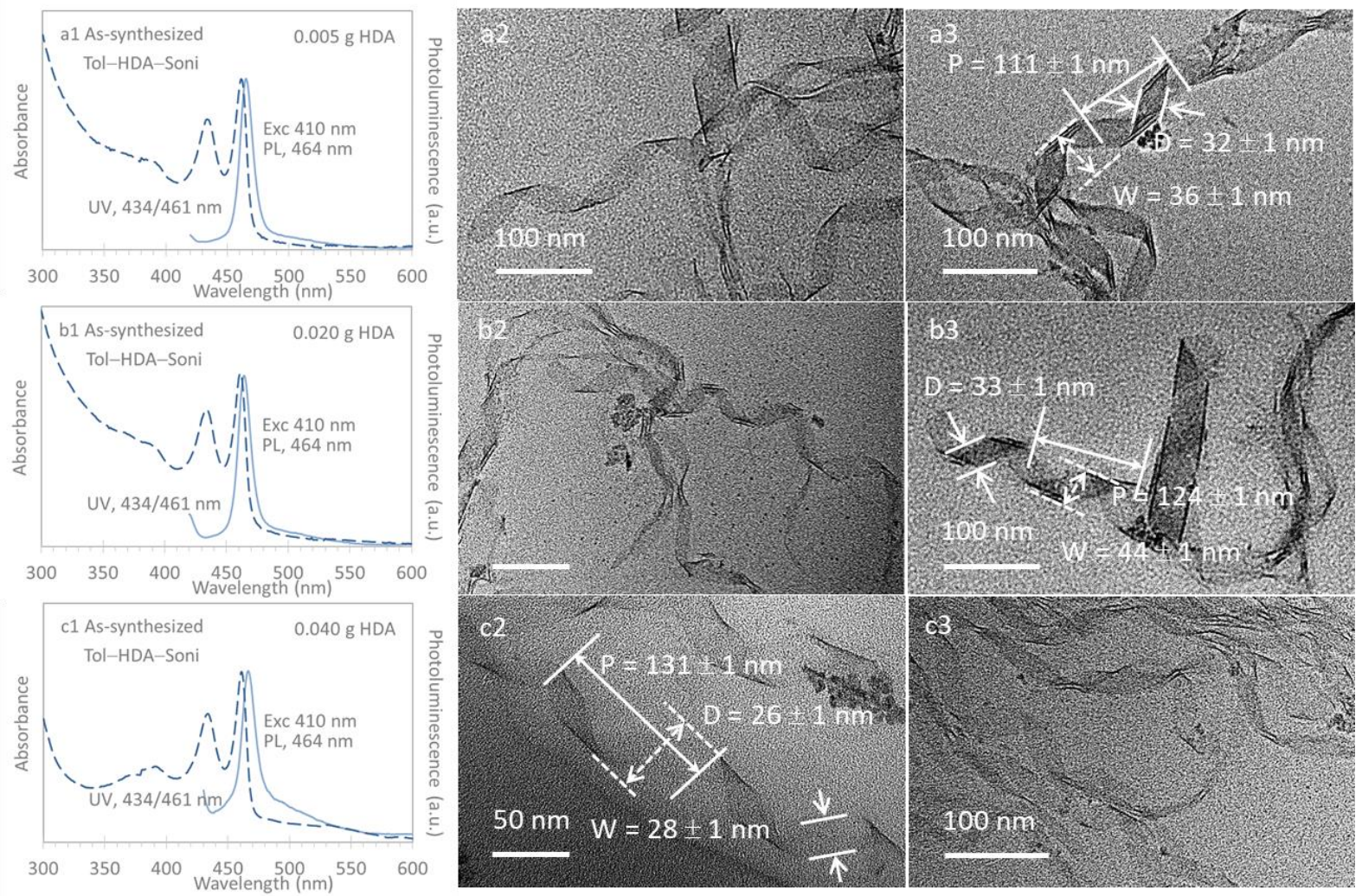

Figure S1-3. The effect of the sonication period explored for the formation of the helical nanostructure. Normalized absorption (dashed traces, left $y$ axis) and emission (solid traces, right $y$ axis) and TEM images of one reaction sample. The sample (15 $\mu \mathrm{L})$ was dispersed in $3.0 \mathrm{~mL}$ of Tol containing 0.005 (a), 0.020 (b), and 0.040 (c) g HDA. The dispersions were sonicated for $30 \mathrm{~min}$, before the optical and TEM measurements. There is large experimental window for the HDA amount, regarding the formation of the helical nanostructure under our experimental conditions. 

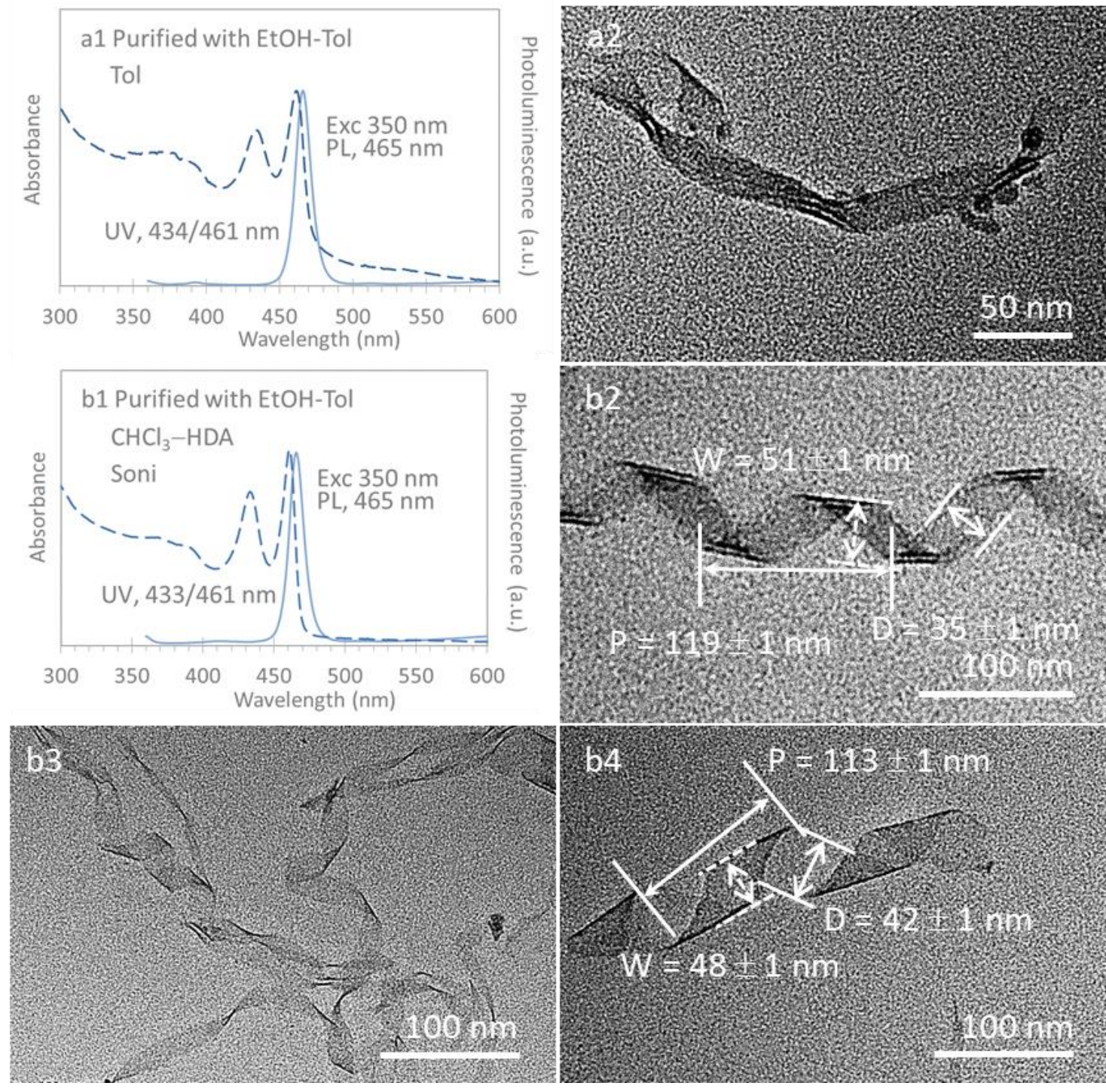

Figure S2-1. Normalized optical properties (a1 and b1) of absorption (dashed traces, left y axis) and emission (solid traces, right y axis) and TEM images (a2 and b2 to b4) collected for our purified sample from a different synthetic batch (other than that shown in Figure 2). The purification was carried out with EtOH and Tol; $15 \mu \mathrm{L}$ of our sample was added into a mixture of $2.0 \mathrm{~mL}$ Tol and $1.0 \mathrm{~mL}$ EtOH. Centrifugation was carried out. After three times of purification, the two resulting purified samples were dispersed in $3.0 \mathrm{~mL}$ of Tol (a) and in 3.0 $\mathrm{mL}$ of $\mathrm{CHCl}_{3}$ containing $0.010 \mathrm{~g}$ of $\mathrm{HDA}(\mathrm{b})$, followed by a $30 \mathrm{~min}$ sonication. 

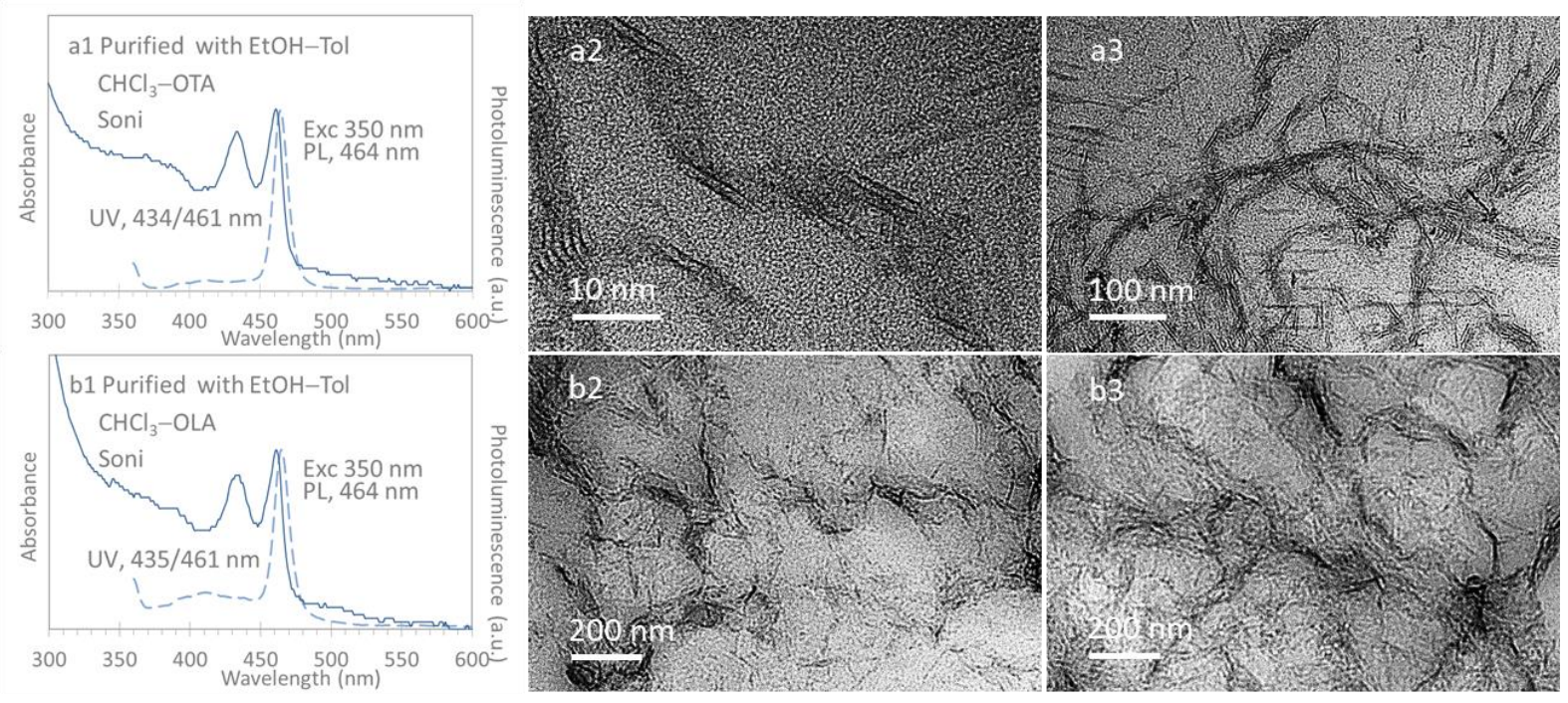

Figure S2-2. Normalized absorption (solid traces, left $y$ axis) and emission (dashed traces, right $y$ axis) spectra and corresponding TEM images of two purified sample dispersions. The samples $(15 \mu \mathrm{L})$ were purified in an identical way, with $3.0 \mathrm{~mL}$ of the Tol and EtOH mixture. After three times of purification, the products were dispersed in $3.0 \mathrm{~mL}$ of $\mathrm{CHCl}_{3}$ containing $0.010 \mathrm{~g}$ of OTA (a) and OLA (b), followed by a $30 \mathrm{~min}$ sonication. For Dispersion a, CdSe helical nanostructures were observed, but of relative poor quality and low yield. For Dispersion b, the helical nanostructures were even fewer. The dispersity of a purified sample is better in $\mathrm{HDA}$-containing $\mathrm{CHCl}_{3}$. 


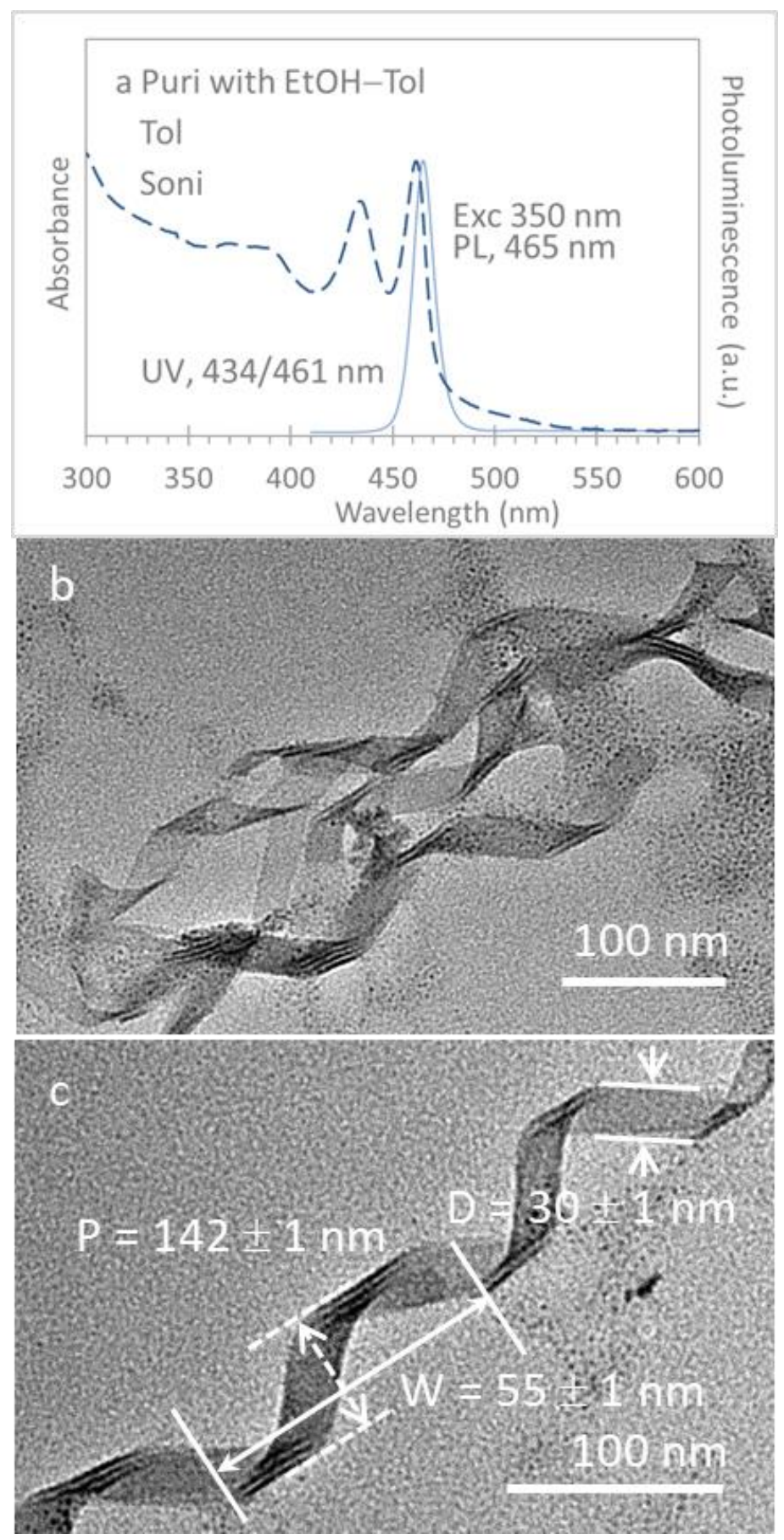

Figure S2-3. Normalized absorption (dashed trace, left y axis) and emission (solid trace, right $y$ axis) and TEM images of another sample purified. The purification was performed after 38day synthesis, with $15 \mu \mathrm{L}$ of our sample in a mixture of $2.0 \mathrm{~mL}$ Tol and $1.0 \mathrm{~mL}$ EtOH.

Centrifugation was carried out. After three times of purification, the sample was dispersed in $3.0 \mathrm{~mL}$ of Tol followed by a $30 \mathrm{~min}$ sonication. Clearly, for the NPL to helical nanostructure transformation, sonication alone works. 

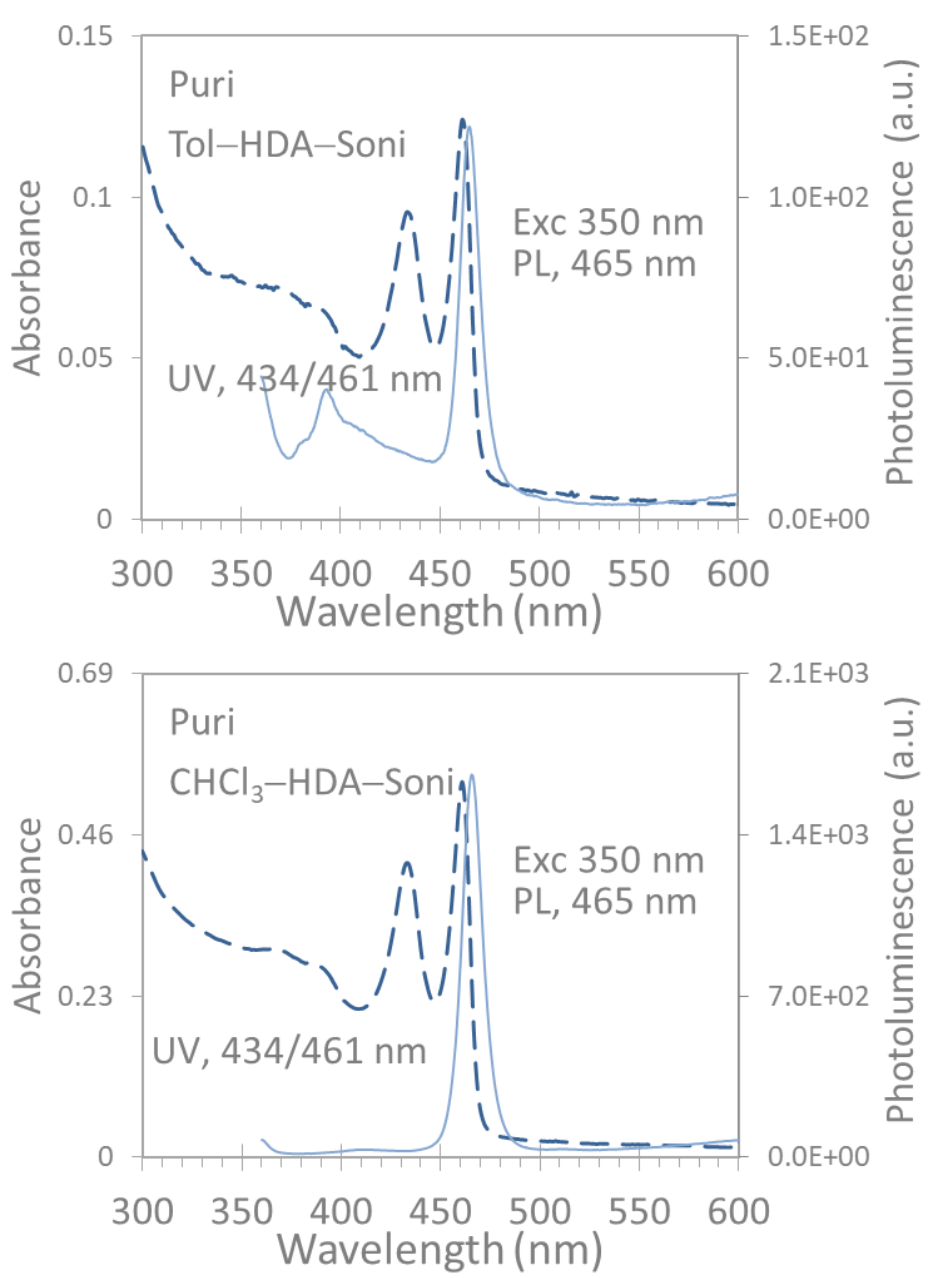

Figure S2-4. Optical properties of one purified sample (15 $\mu \mathrm{L}$ by to $2 \mathrm{Tol}-1 \mathrm{EtOH}$ three times) to illustrate the fact that the dispersity in $\mathrm{HDA}$-containing $\mathrm{CHCl}_{3}$ is better than that in $\mathrm{HDA}$ containing Tol. The effect of the dispersions on the formation of CdSe nanohelices from the purified CdSe NPLs might be different from that documented. ${ }^{1}$ The Sci Adv paper deals with the addition of oleic acid (OA) to induce the self-assembly of CdSe nanoplatelets (NPLs) into chiral ribbons with a pitch of $\sim 400 \mathrm{~nm}$ and the length of several micrometers. It was claimed that as the ligand coverage increased, isolated NPLs in dispersion became twisted. Regarding the formation of one NPL and its transformation into one nano-helix, particularly during a sample incubation in our case, it might be related to surface ligands also.

1 Jana, S.; Frutos, M. d.; Davidson, P.; Abécassis, B. Ligand-Induced Twisting of Nanoplatelets and Their Self-Assembly into Chiral Ribbons. Sci. Adv. 2017, 3, e1701483. 


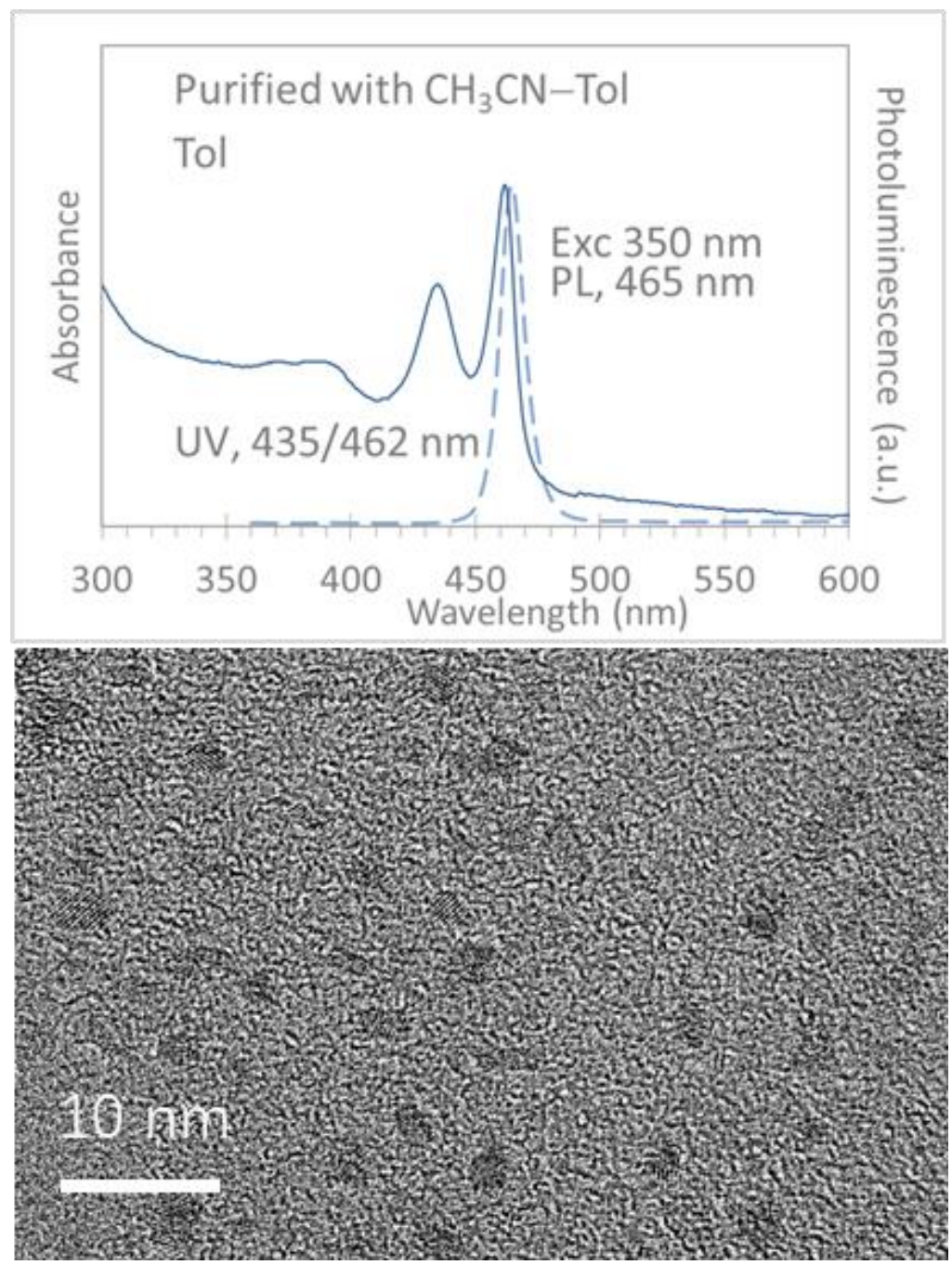

Figure S3. Normalized absorption (solid trace, left $y$ axis) and emission (dashed trace, right $y$ axis) spectra and one corresponding TEM image of our sample (15 $\mu \mathrm{L}$, synthesized from a different batch other than that shown in Figure 3), which was purified with $2.0 \mathrm{~mL}$ Tol -1.0 $\mathrm{mL} \mathrm{CH}{ }_{3} \mathrm{CN}$ (three times), and dispersed in $3.0 \mathrm{~mL}$ of Tol. The OD MSCs are relatively undisturbed. 


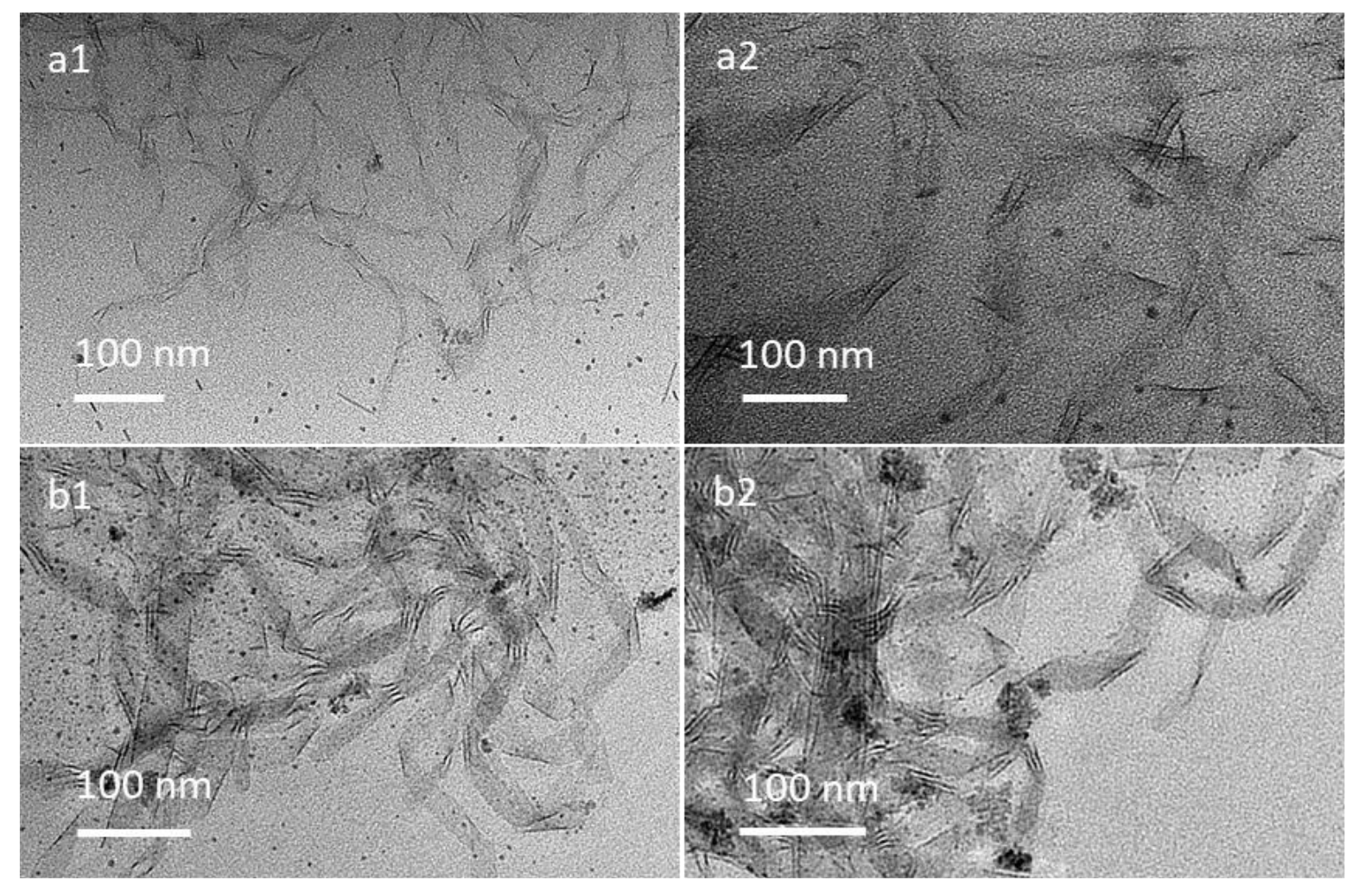

Figure S4-1. TEM images of two samples (15 $\mu \mathrm{L}$ each) incubated without purification for 7 (a) and 38 (b) days (in storage after synthesis). The samples were dispersed in $3.0 \mathrm{~mL}$ of Tol containing $0.010 \mathrm{~g}$ of HDA. No sonication was performed. Both 2D NPLs and helical nanostructures are observed, although the former dominates. It seems that HDA is able to assist the formation of 2D NPLs for incubated samples in Tol. 

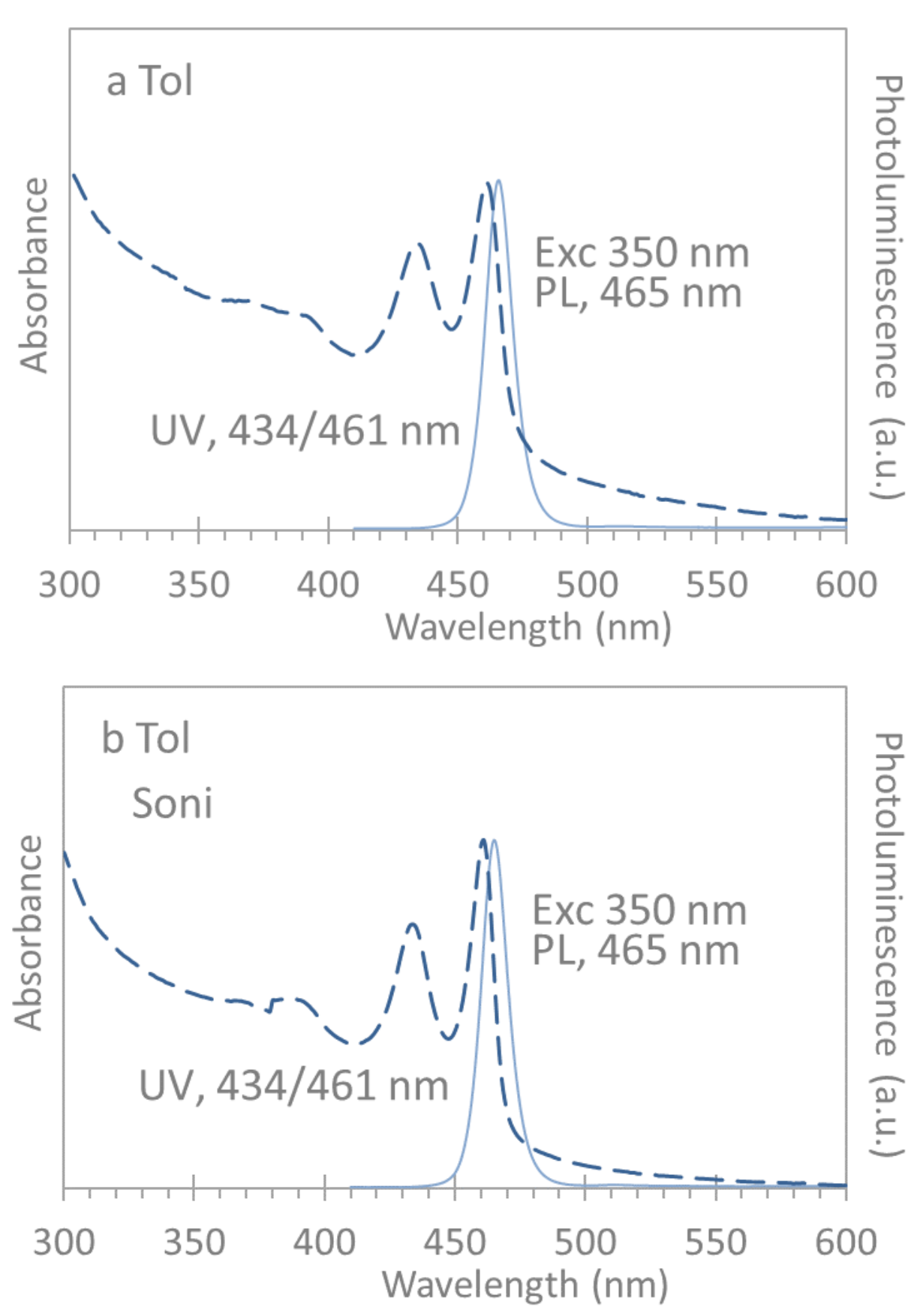

Figure S4-2. Normalized absorption (dashed traces, left $y$ axis) and emission (dashed traces, right $y$ axis) spectra of Dispersions a and b used for Figure 4. 


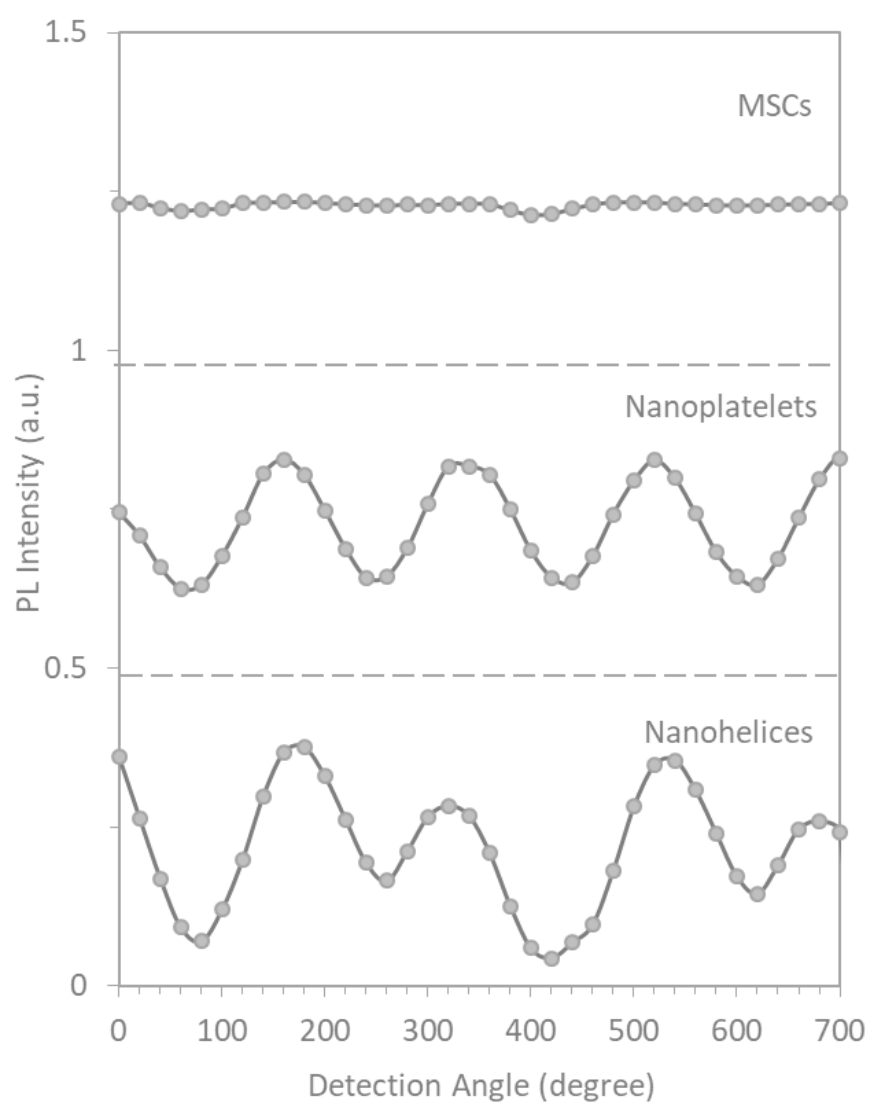

Figure S5. Preliminary results of polarized emission (Horiba Fluorolog, excited at $430 \mathrm{~nm}$ ) for the CdSe MSCs, nanoplatelets and nanohelices. The spectra (offset), showing PL intensity as a function of detection angle, were collected by dispersed one as-synthesized sample (15 $\mu \mathrm{L})$ in $3.0 \mathrm{~mL}$ of Tol (top), one sample $(15 \mu \mathrm{L})$ purified with $2.0 \mathrm{~mL}$ Tol $-1.0 \mathrm{~mL} \mathrm{CH}{ }_{3} \mathrm{CN}$ and dispersed in $3.0 \mathrm{~mL}$ of Tol for $3 \mathrm{~h}$ to resulting in 2D NPLs (middle), and one sample (15 $\mu \mathrm{L}$ ) purified with $2.0 \mathrm{~mL}$ Tol $-1.0 \mathrm{~mL} \mathrm{EtOH}$ and dispersed in $3.0 \mathrm{~mL}$ of $\mathrm{CHCl}_{3}$ containing $0.010 \mathrm{~g}$ HDA with a 30 min sonication to result in 2D helical nanostructures (bottom). Two $\lambda / 4$ circular polarizers were placed before and before our sample holder, with the excitation wavelength of $430 \mathrm{~nm}$ and the scanning range of 440 to $500 \mathrm{~nm}$. 0 to 360 degrees is sufficient for the plots, although here we plotted 0 to 700 degrees; we rotated the polarizer once every twenty degrees. The OD MSCs do not exhibit an angle anisotropy, while the 2D NPLs and 2D nanohelices do. Furthermore, the 2D NPLs show a sinusoidal variation of intensity with the detection angle, while the 2D nanohelices display an asymmetric sinusoidal change. The polarized emission demonstrates that the OD MSCs, 2D NPLs, and 2D nanohelices have different structural anisotropies. We thank Mr. Long Xu (from Horiba in Chengdu) for useful discussions. 